\title{
DESCRIÇÃO DA FARMACOTERAPIA DA CROMOBLASTOMICOSE: REVISÃO DA LITERATURA
}

\author{
DESCRIPTION OF CHROMOBLASTOMYCOSIS PHARMACOTHERAPY: \\ LITERATURE REVIEW
}

Maria de Fátima Souza Costa $^{1}$, Lidiane Rodrigues Monteiroํㅜ Ana Beatriz Silva de Moura ${ }^{1}$, Daniella Paternostro de Araújo Grisóliaª

1 Universidade da Amazônia E-mail para correspondência: daniellapaternostro@gmail.com Submetido em: 06/11/2021 e aprovado em: 10/01/2022

\begin{abstract}
RESUMO
Introdução: A cromoblastomicose (CBM) é uma micose por implantação fúngica na pele, de evolução crônica, causada pela inoculação traumática de fungos dematiáceos. Objetivos: identificar a partir de uma análise na literatura os antifúngicos e as associações terapêuticas mais utilizados no tratamento, assim como, determinar as doses eficazes. Métodos: a pesquisa foi realizada a partir de uma revisão integrativa, com busca eletrônica de artigos nas bases de dados SCIELO, PUBMED, BVS e CINHHL através de descritores, "farmacoterapia da CBM", "doses e associações terapêuticas" no período de 2011 a 2021. Resultados e discussão: os principais antifúngicos utilizados no tratamento da CBM foram: Itraconazol (ITZ) em doses terapêuticas de 200 a 400 mg/dia, Terbinafina (TBF) de 150 a 1000 mg/dia e Posaconazol (PCZ) 400 a $800 \mathrm{mg} /$ dia. Nas combinações antifúngicas, observou-se que ITZ + TBF foi a mais sugerida entre os artigos avaliados. Os dados mostraram que o ITZ e TBF foram utilizados por um período de 6 a 12 meses com boa resposta clínica. O PCZ pode ser uma opção de tratamento nas formas graves e refratárias, apesar de seu uso nesta patologia ainda se encontra limitado devido ao seu alto custo. Conclusão: CBM é uma micose negligenciada e difícil tratar, especialmente nas formas clínicas mais graves. O ITZ entre os antifúngicos estudados é o mais eficaz tanto em teste de suscebilidade in vitro como em situações clínicas. A interação medicamentosa entre ITZ+TBF é a mais eficiente entre os artigos avaliados na literatura, tornando uma alternativa terapêutica.
\end{abstract}

Palavras-chave: Cromoblastomicose, antifúngicos, associações terapêuticas, suscetibilidade antifúngica.

\begin{abstract}
Introduction: Chromoblastomycosis (CBM) is a chronic mycosis caused by fungal implantation in the skin, caused by the traumatic inoculation of dematiaceous fungi. Objectives: to identify, based on literature review, the most used antifungal agents and therapeutic associations in the treatment, as well as to determine the effective doses. Methods: integrative literature review performed in the electronic bibliographic databases SCIELO, PUBMED, BVS and CINHHL, using descriptors, "CBM pharmacotherapy", "doses and therapeutic associations" between 2011 and 2021. Results and discussion: the main antifungal agents used in the treatment of
\end{abstract}


CBM were: Itraconazole (ITZ) at therapeutic doses from 200 to $400 \mathrm{mg} /$ day, Terbinafine (TBF) from 150 to $1000 \mathrm{mg} /$ day and Posaconazole (PCZ) 400 to $800 \mathrm{mg} / \mathrm{day}$. In antifungal combinations, it was observed that ITZ + TBF was the most suggested among the evaluated articles. Data showed that ITZ and TBF were used for a period of 6 to 12 months with good clinical response. PCZ can be a treatment option for severe and refractory forms, although its use in this pathology is still limited due to its high cost. Conclusion: CBM is a neglected and difficult to treat mycosis, especially in the most severe clinical forms. Among the antifungal agents studied, ITZ is the most effective both in in vitro susceptibility testing and in clinical situations. The drug interaction between ITZ+TBF is the most efficient among the articles evaluated in the literature, making it a therapeutic alternative.

Keywords: Chromoblastomycosis, antifungals, therapeutic combinations, antifungal susceptibility.

\section{INTRODUÇÃO}

A cromoblastomicose (CBM) é uma infecção fúngica crônica, definida como uma micose por implantação na pele, causada pela inoculação traumática de fungos dematiáceos ou melanizados, pertencentes à família Herpotrichiellaceae ${ }^{(1)}$. Os membros desta família são caracterizados por apresentarem coloração variável de verde escuro à marrom e negro devido à presença de melanina na parede celular. Essas, por sua vez, são importantes por estarem associadas com ação fotoprotetora, que permite ao fungo se desenvolver nos ambientes ensolarados, e também atua como fator de virulência reduzindo a suscetibilidade fúngica aos mecanismos de defesa do hospedeiro ${ }^{(2,3,4)}$.

Em 1911, o primeiro caso de CBM foi observado por Alexandrino Pedroso ao visualizar presença de corpos escleróticos em um paciente de São Paulo, Brasil. O médico alemão Max Rudolph publicou os primeiros casos da doença em 1914, ao isolar fungos de cor escura a partir de quatro pacientes, denominando esta doença com o nome de "figueira" em trabalhadores rurais oriundos do estado de Minas Gerais e São Paulo ${ }^{(5)}$. Em 1922, Terra e colaboradores definiram a doença como uma dermatite verrucosa causada por fungos pigmentados e estabeleceram o nome de "cromoblastomicose". Esta doença também é conhecida como: formigueiro, blastomicose negra, cromomicose e pé-musgoso ${ }^{(6,7,8)}$.

A CBM é considerada uma doença ocupacional, ocorrendo principalmente em trabalhadores rurais, madeireiros ou vendedores de produtos agrícolas, que não utilizam equipamentos de proteção individual quando manuseiam o solo, vegetação e plantas em decomposição ${ }^{(9)}$. Diante destes dados, considera-se como estratégia de prevenção melhoria das condições de trabalho de lavradores por meio da utilização de calçados e vestuários apropriados, além de treinamento em saúde sobre os riscos associados as suas atividades diárias ${ }^{(1)}$. 
Os sintomas consistem em prurido de intensidade variável, dor e presença de lesões que se localizam geralmente, nos membros inferiores e raramente em outros locais da superfície cutânea ${ }^{(2,7)}$. Esta doença pode afetar qualquer idade, mas raramente ocorre antes da idade adulta e predomina em homens de 30 a 50 anos, o que se justifica pelo fato de que os traumatismos que propiciam a implantação transcutânea do fungo resultam das atividades rurais, as quais são exercidas principalmente pelos homens ${ }^{(10)}$.

Estudos epidemiológicos demonstram que a CBM tem distribuição mundial, descrita nas Américas, Europa, Ásia, Oceania e África, com alta prevalência nas regiões de clima tropicais e subtropicais. No Brasil, mais de 500 casos de CBM já foram documentados na literatura. No entanto, acredita-se que existam mais casos desta doença, uma vez que não é uma doença de notificação compulsória ${ }^{(11)}$. Esta micose ocorre em vários estados, com casos distribuídos em todas as regiões geográficas. Vale ressaltar que estado do Pará é a principal área endêmica da CBM no Brasil, com 325 casos registrados entre os anos de 1942 e 1997, sendo a maior parte dos pacientes moradores da região nordeste do estado ${ }^{(12)}$.

Os agentes etiológicos mais comuns pertencem aos gêneros Cladophialophora e Fonsecaea. Cladophialophora carrionii é o patógeno predominante em regiões secas e semidesérticas da Austrália, África do Sul, Venezuela e Cuba, enquanto Fonsecaea pedrosoi é o agente mais comum encontrado em zonas tropicais úmidas, como Amazônia e regiões temperadas da América Latina ${ }^{(13)}$. Outros fungos dematiáceos têm sido associados com menor frequência a CBM, entre eles temos: Phialophora verrucosa, Rhinocladiella aquaspersa, Exophiala dermatitidis, Exophiala jeanselmei e Exophiala spinifera ${ }^{(14,15)}$.

De acordo com processo fisiopatológico da CBM, a infecção ocorre após traumatismo da pele e inoculação traumática de fungos dematiáceos ${ }^{(16)}$. No local da implantação se forma uma lesão unilateral, com a presença de pequenas pápulas eritematosas de superfície lisa. As pápulas aumentam de tamanho em poucas semanas, algumas vezes apresentando superfície escamosa. Com o passar do tempo, a lesão evolui para diferentes tipos de lesões, caracterizando o aspecto clínico polimórfico da doença. O curso crônico inicia-se com lesões verrucosas associadas a nódulos, estes podem ulcerar e tornar-se vegetantes, com aspecto papilomatoso semelhante à couve-flor $^{(1,8)}$. Entre as complicações observadas na CBM incluem: infecções bacterianas secundárias, elefantíase e carcinoma de células escamosas ${ }^{(16,17,18)}$.

Em 1950, Carrión introduziu uma classificação clínica para as lesões de CBM definida em cinco diferentes formas: nodular, tumoral, verrucosa, placa e cicatricial. Posteriormente, 
alguns pesquisadores passaram a classificar as lesões de acordo com o grau de severidade: formas leves, moderadas e severas, baseadas na extensão da área atingida, número de lesões, presença de complicações e resposta ao tratamento ${ }^{(1,8)}$. Salgado e colaboradores em 2005 descreveram uma nova forma clínica, CBM cutânea difusa, caracterizada por lesões nodulares e verrucosas com extensa distribuição na pele. Quatro anos depois, este mesmo grupo publicou casos de lesões localizadas, papuloescamosas, com formato anular denominada de CBM cutânea localizada anular ${ }^{(19,20)}$. Esses fatos levaram a uma nova proposta de classificação baseada no aspecto que as lesões assumem ao longo da evolução da doença, como observado na Figura 1.

Figura 1: Classificação da CBM de acordo com o aspecto das lesões. A CBM pode ser dividida em dois grupos: CBM cutânea localizada, que pode ser: (A) Anular, (B) Em placa (C) Nodular e CBM cutânea difusa (D).

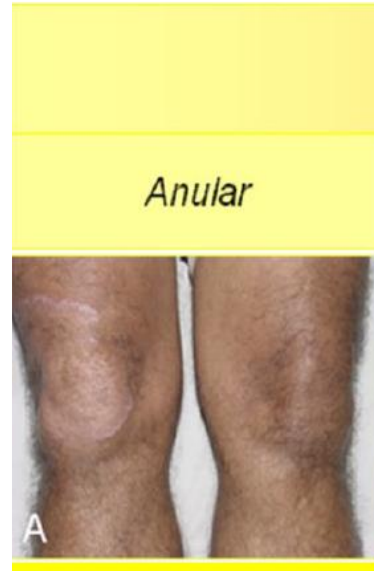

6 a 12 meses

\section{Cutânea localizada}

Em placa

B

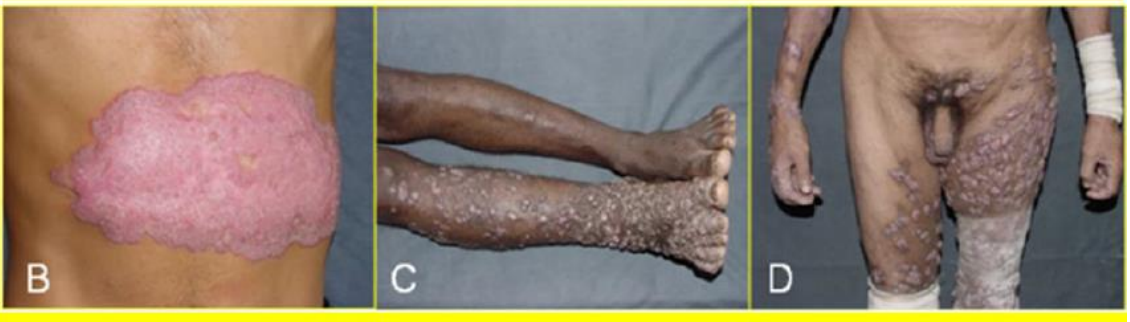

9 meses a mais que 3 anos
Cutânea

Difusa

Fonte: Salgado, 2005/2009.

O diagnóstico desta micose pode ser realizado através do exame micológico direto, a partir do raspado das lesões na pele, onde são observadas as células escleróticas, que são similares para os diferentes agentes etiológicos, e caracterizam-se por divisão multisseptada, parede espessa, cor acastanhada devido a produção de melanina na parede celular ${ }^{(8,16)}$. Posteriormente este fungo quando cultivado em meio Ágar Sabouraud apresenta colônia de aspecto aveludado, anverso com coloração variando entre o verde-oliva e o negro, sendo o reverso também enegrecido, porém estas características macroscópicas não caracterizam o agente etiológico, pois diferentes espécies apresentam o mesmo aspecto. A identificação dos agentes etiológicos tem sido feita por meio do microcultivo em lâmina, podendo-se inferir as espécies de acordo com o tipo de conidiação mais prevalente ${ }^{(17)}$.

O tratamento da CBM é um dos mais difíceis e várias terapias têm sido utilizadas, pois não existe um tratamento padronizado para esta doença ${ }^{(13)}$. A terapia é longa e sujeita a 
recidivas, e dependendo da situação clínica e da extensão das lesões, os procedimentos terapêuticos podem $\operatorname{variar}^{(18)}$. No Brasil, esta infecção fúngica não é de notificação, portanto não se dispõe de dados precisos sobre sua incidência; o conhecimento de áreas endêmicas, dados de prevalência, incidência e morbidade. Alguns medicamentos antifúngicos para o tratamento estão disponíveis no Sistema Único de Saúde (SUS) e são oferecidos gratuitamente. Entre eles estão: itraconazol, terbinafina, cetoconazol, fluconazol entre outros ${ }^{(20)}$.

Entre os métodos terapêuticos utilizados destaca-se: farmacoterapia, terapias físicas e combinação de ambas. Na farmacoterapia, diferentes esquemas terapêuticos, com o uso da anfotericina B, 5-fluorcitosina, fluconazol, tiabendazol e cetoconazol, foram utilizados nas últimas décadas, entretanto com baixa taxa de cura ${ }^{(21,7)}$. Quanto aos métodos físicos, incluem a termoterapia, crioterapia e a terapia fotodinâmica, podendo ainda ser utilizadas cirurgias convencionais ou a laser, indicadas em casos de lesões iniciais ou de pequena extensão ${ }^{(2,6)}$.

Associações terapêuticas têm apresentado bons resultados, como por exemplo, os sinergismos entre itraconazol e terbinafina. anfotericina-B e 5-fluorcitosina, 5-fluorcitosina e Itraconazol $^{(7,22)}$. Entretanto, o uso associado de antifúngicos também pode apresentar algumas desvantagens, como por exemplo: aumento do risco de aparecimento de reações adversas aos medicamentos (RAM) e interações medicamentosas desfavoráveis, portanto, devem ser realizados com cautela ${ }^{(23)}$. Neste contexto, os testes de suscetibilidade antifúngica in vitro são considerados a melhor estratégia para a seleção do fármaco mais apropriado ${ }^{(24)}$.

Os pacientes com CBM representam um verdadeiro desafio terapêutico devido sua natureza recalcitrante, especialmente nas formas clínicas graves que quando não tratadas de forma adequada pode levar o paciente uma incapacidade para o trabalho além de custos sociais e problemas psicológicos ${ }^{(21)}$.

Diante disso do exposto e com a preocupação referente ao tratamento da CBM, este estudo busca a partir de uma revisão integrativa identificar os principais fármacos utilizados, além de descrever os mais ativos e as doses terapêuticas eficazes e por último determinar as combinações de antifúngicos utilizadas.

\section{MÉTODOS}

O presente artigo foi realizado a partir de uma revisão integrativa, uma nova abordagem metodológica que é capaz de proporcionar através de estudos experimentais e não experimentais informações mais amplas sobre um determinado problema e/ou assunto, 
resultando assim em um corpo de conhecimento. O trabalho foi desenvolvido com base na principal pergunta norteadora: "Quais são os medicamentos utilizados no tratamento da CBM?”; seguido das perguntas secundárias: “Quais os antifúngicos mais ativos e menos ativos usados na CBM? e "Qual é a dose mais eficiente para entre os fármacos utilizados para o tratamento da CBM?" e "Quais as principais associações terapêuticas utilizados para o tratamento da CBM?"

Para levantamento dos artigos na literatura foram utilizados por meio de busca eletrônica trabalhos científicos nas bases de dados do BVS, CINHHL, PUBMED e SCIELO. A busca por artigos foi realizada pela combinação das seguintes combinações de palavras-chaves de acordo com o assunto em questão: "CBM", "farmacoterapia da CBM", "doses e associações terapêuticas na CBM", "suscetibilidade antifúngica"; sendo inseridas as quatro palavras-chaves em cada base, sem utilização de termos truncados ou operadores booleanos.

Foram estabelecidos para a obtenção dos resultados fatores de inclusão e exclusão para a análise da temática proposta. Os critérios de inclusão, foram: a) Publicação entre o período de 2011 a 2021, b) língua inglesa e espanhola c) responder os objetivos proposto no trabalho. No que se diz respeito aos critérios de exclusão: e) não foram utilizadas monografia e dissertações, f) arquivos que não estavam disponíveis na integra g) artigos repetidos.

Após a realização da busca em cada base de dados, encontrou-se 627 artigos no total, e 40 artigos corresponderam aos critérios de inclusão/exclusão estabelecidos para a pesquisa. Posteriormente, seguiu-se com a análise dos artigos pelo título para análise de duplicata ou ainda relação direta com as questões norteadoras, enquadrando-se apenas 21 artigos com esses quesitos, eles foram numerados de 1 a 21 na sequência que foram encontrados e, prosseguiu-se com a extração dos achados que respondessem de modo qualitativo às perguntas norteadoras, não importando a metodologia aplicada no estudo, conforme descrito na Figura 2.

Figura 2: Fluxograma de identificação, seleção e inclusão dos artigos, para desenvolvimento do estudo.

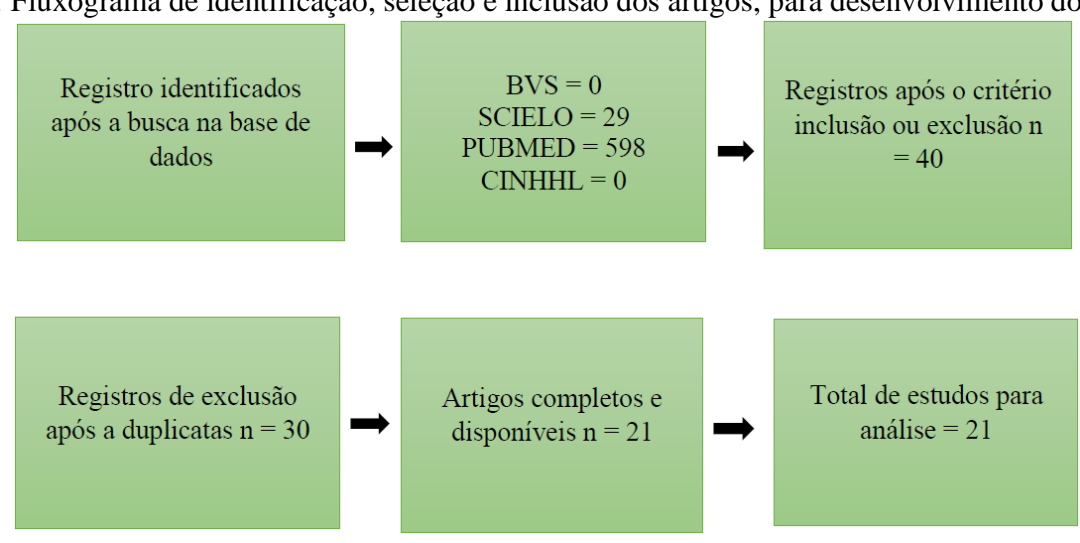

Fonte: Autores, 2021. 


\section{RESULTADOS}

Após o levantamento realizado na literatura científica sobre a farmacoterapia da CBM foram selecionados 21 artigos entre os anos de 2011 a 2021 conforme a Tabela 1.

Tabela 1 - Artigos selecionados com relação ao tratamento farmacológico da CBM.

\begin{tabular}{|c|c|c|}
\hline Autor(es) & Ano de publicação & Título do estudo \\
\hline Krzysciak et al., & 2014 & Chromoblastomycosis \\
\hline Chowdhary et al., & 2014 & $\begin{array}{l}\text { ESCMID and ECMM joint clinical guidelines for the } \\
\text { diagnosis and management of systemic } \\
\text { phaeohyphomycosis: diseases caused by black } \\
\text { fungi. }\end{array}$ \\
\hline Sousa et al., & 2014 & $\begin{array}{l}\text { Topical application of imiquimod as a treatment for } \\
\text { chromoblastomycosis. }\end{array}$ \\
\hline Chaidaroon et al., & 2015 & $\begin{array}{l}\text { Corneal Chromoblastomycosis Caused by } \\
\text { Fonsecaea pedrosoi. }\end{array}$ \\
\hline Queiroz-Telles & 2015 & Chromoblastomycosis: a neglected tropical disease. \\
\hline Sophie et al., & 2015 & $\begin{array}{l}\text { First case of chromoblastomycosis from } \\
\text { Bangladesh. }\end{array}$ \\
\hline Lima et al., & 2016 & Response of chromoblastomycosis to voriconazole. \\
\hline Carrasco-Zuber et al., & 2016 & $\begin{array}{l}\text { Cutaneous Involvement in the Deep Mycoses: A } \\
\text { Literature Review. Part I-Subcutaneous Mycoses. }\end{array}$ \\
\hline Sienkiewicz et al., & 2016 & $\begin{array}{l}\text { Comparison of clinical pharmacology of } \\
\text { voriconazole. }\end{array}$ \\
\hline Agarwal et al., & 2017 & $\begin{array}{l}\text { Chromoblastomycosis in Índia: Review of } 169 \\
\text { cases. }\end{array}$ \\
\hline Queiroz-Telles et al., & 2017 & Chromoblastomycosis. \\
\hline Heidrich & 2017 & $\begin{array}{l}\text { Chromoblastomycosis caused by Rhinocladiella } \\
\text { similis: Case report. }\end{array}$ \\
\hline Rio et al., & 2017 & $\begin{array}{l}\text { Chromomycosis, na unusual cause of cicatricial } \\
\text { ectropion: a case report. }\end{array}$ \\
\hline Tourret et al., & 2017 & $\begin{array}{l}\text { Unique case report of a chromomycosis and Listeria } \\
\text { in soft tissue and cerebellar abscesses after kidney } \\
\text { transplantation. }\end{array}$ \\
\hline Ventura-Flores et al., & 2017 & $\begin{array}{l}\text { Chromoblastomycosis: clinical and microbiological } \\
\text { characteristics of a neglected disease }\end{array}$ \\
\hline Brito et al., & 2017 & $\begin{array}{l}\text { Chromoblastomycosis: an etiological, } \\
\text { epidemiological, clinical, diagnostic, and treatment } \\
\text { update. }\end{array}$ \\
\hline Deng et al. & 2018 & Combination of Amphotericin B and Terbinafine \\
\hline
\end{tabular}






Identificamos que os antifúngicos mais utilizados foram: Itraconazol (ITZ) com 16 citações, Terbinafina (TBF) com 15 citações, Posaconazol (PCZ) com 10 citações, Anfotericina-B (ANF) com 7 citações, Cetoconazol (CTZ) com 3 citações, Fluconazol (FCZ) com 3 citações, Ravuconazol (RVC) com 3 citações, Fluocitosina (5-FLU) com 3 citações ,Tiabendazol (TBZ) com 2 citações, Isavuconazol (ISA) com 2 citações, Caspofungina (CAS) com 2 citações, Micafungina (MIA) com 2 citações e Anidulafungica (ANI) com 2 citações (Figura 2A).

Com objetivo de determinar os antifúngicos com melhor desempenho no tratamento da CBM dividimos em dois grupos com maior e menor atividade contra agente etiológico da doença e a resposta terapêutica (Figura 2B). O primeiro grupo formado por ITZ, TBF, RVC, PCZ e VCZ identificou-se os antifúngicos mais ativos ${ }^{(7,8,13,15,16)}$. No segundo grupo constituído por ANF, 5-FLU, ANI, ISA, FCZ e CTZ identificou-se os antifúngicos menos ativos ${ }^{(6,24,25,26)}$. 
Figura 3: Perfil de fármacos utilizados no tratamento da CBM. A figura 3A apresenta os fármacos mais citados para o tratamento da CBM. A figura 3B demonstra os antifúngicos mais e menos ativos utilizados no tratamento da CBM nos últimos 10 anos. Itraconazol (ITZ), Terbinafina (TBF), Posaconazol (PCZ), Anfotericina-B (ANF), Cetoconazol (CTZ) Fluconazol (FCZ), Ravuconazol (RVC), Fluocitosina (5-FLU), Tiabendazol (TBZ), Isavuconazol (ISA), Caspofungina (CAS), Micafungina (MFG) e Anidulafungica (ANI).
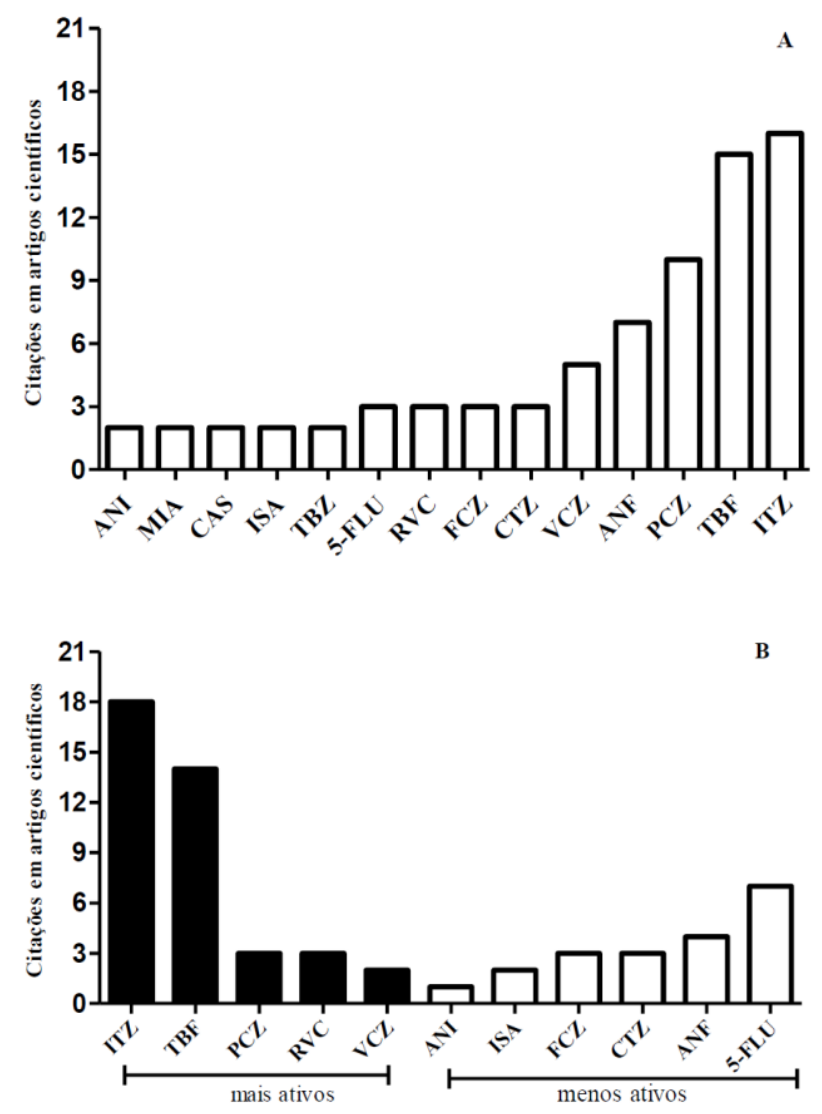

Fonte: Os autores, 2021.

Após os estudos realizados sobre os antifúngicos mais ativos no tratamento da CBM, foram analisadas quais doses terapêuticas obtiveram melhores resultados clínicos aos pacientes para o ITZ as doses terapêuticas variam de 200 a 400 mg/dia, TBF 150 a 1000 mg/dia, PCZ 400 a 800 mg/dia, RVC e VCZ 200 mg/dia.

Avaliamos também as combinações terapêuticas mais utilizadas em situações onde os pacientes apresentam resistência ao tratamento. Entre as associações, observou-se que a combinação entre os antifúngicos ITZ + TBF (com 10 citações) e ANF + 5-FLU (com 8 citações) foram as mais sugeridas entre os artigos estudados conforme descrito na figura 4. 
Figura 4: Determinação das combinações antifúngicas realizadas no tratamento da CBM nos últimos 10 anos. ANF+TBF (Anfotericina B + Terbinafina), ANF + FCZ (Anfotericina B + Fluconazol), PCZ + 5-FLU (Posaconazol + 5-Fluocitosina), ITZ+ 5-FLU (Itraconazol+5-Fluocitosina), ANF+5-FLU (Anfotericina B+ 5-Fluocitosina), ITZ+TBF (Itraconazol+Terbinafina).



Fonte: Os autores, 2021.

\section{DISCUSSÃO}

A CBM é uma doença de difícil tratamento, em parte pela falta de padronização do esquema terapêutico resultado da utilização de várias opções de tratamento, com baixas taxas de cura e frequentes recidivas ${ }^{(23)}$. A escolha do tratamento e os resultados dependem do agente etiológico, tamanho e extensão das lesões e presença de complicações ${ }^{(8)}$. A terapia com os antifúngicos consiste em períodos longos, muitas vezes combinações entre fármacos são utilizados para casos graves desta micose ${ }^{(19,23)}$. Diante desta realidade, o presente estudo procurou descrever os antifúngicos mais eficazes e as associações terapêuticas com melhores resposta ao tratamento.

Entre os quatorze antifúngicos identificados cinco apresentaram maior eficácia para o tratamento da CBM como: VCZ, PCZ, RVC, TBF e ITZ. Estes dados concordam com os estudos de Queiroz-Telles e colaboradores (2017), que mostraram que o ITZ (200 - $400 \mathrm{mg} / \mathrm{dia})$ e TBF (500 - $1000 \mathrm{mg} / \mathrm{dia}$ ) foram utilizados por um período de 6 a 12 meses com boa resposta clínica e atividade in vitro contra os agentes causadores de CBM. A taxa de cura de ITZ variam de 15 a $80 \%^{(2)}$. Apesar desta micose não ter um tratamento padronizado, observamos que 16 artigos mencionaram o ITZ como uma boa opção, isso pode estar relacionado ao seu mecanismo de ação que consiste na inibição $14 \alpha$-desmetilase responsável por fazer a conversão do lanosterol em ergosterol um componente vital pra célula fúngica ${ }^{(27)}$.

O segundo antifúngico mais utilizado para o tratamento é a TBF a qual possui taxas de cura semelhantes ao ITZ. Este fármaco quando administrado por via oral possui efeitos 
fungistáticos e fungicidas através da inibição da esqualeno-epoxidase, que interfere na biossíntese de ergosterol e função da membrana fúngica ${ }^{(28,29)}$. Estes fármacos são considerados seguros em casos de tratamento prolongado como $\mathrm{CBM}^{(14)}$.

Nos últimos anos o arsenal antifúngico foi ampliado com a inclusão de triazóis de segunda geração como o VCZ, PCZ, RVC que vêm demonstrando eficácia em teste de sensibilidade in vitro e em consequência disso já estão sendo utilizados na terapia da CBM com bons resultados clínicos ${ }^{(30)}$. O PCZ e VCZ pode ser uma opção de tratamento para pacientes que não respondem positivamente a terapia com ITZ ${ }^{(9)}$, mas reações adversas como distúrbios visuais, reações cutâneas, náuseas, vômitos, diarreia, foram observadas, apesar de seu uso nesta patologia ainda se encontra limitado devido ao seu alto custo ${ }^{(3,4,9)}$.

O grupo representado pelos antifúngicos ANI, ISA, FCZ, CTZ, ANF, 5-FLU demonstrou baixa atividade no tratamento da CBM. Atualmente o CTZ não é utilizado na terapia da CBM devido à sua baixa eficiência e elevado risco de efeitos adversos em tratamentos mais longos, como é o caso desta micose. ANF é considerado ineficaz como monoterapia e confirmado em teste de sensibilidade in vitro com altos valores de concentração inibitória mínima ${ }^{(28)}$. Este fármaco é pouco utilizado na terapia devido ao elevado risco de reações adversas, tais como: hepatoxicidade, nefrotoxidade, mielotoxicidade e ginecomastia ${ }^{(8)}$. Nós também avaliamos ANI, ISA, FCZ e 5-FLU, e para esses antifúngicos encontramos resistência nos isolados clínicos, confirmando dados semelhantes obtidos por outros autores ${ }^{(7,16,24)}$.

Em relação a dose terapêutica o ITZ é eficaz de 200 a $400 \mathrm{mg} /$ dia por período de 6 a 12 meses. Também tem sido empregado com sucesso na pulsoterapia que consiste na administração de $400 \mathrm{mg} /$ dia uma vez por semana durante um período estabelecido. Essa terapia reduz a quantidade do medicamento e o custo $^{(13,23)}$. Já TBF na dose de 150 a $1000 \mathrm{mg} / \mathrm{dia}$ durante 12 meses demonstrou boa atividade antifúngica in vitro contra agentes etiológicos e em doses maiores de 500 a $1000 \mathrm{mg} /$ dia tem ação antifibrótica com alto grau de eficácia e tolerabilidade ${ }^{(4)}$.

O PCZ e VCZ em doses variando de 400 a $800 \mathrm{mg} /$ dia é a melhor opção para o tratamento de todas as apresentações clínicas de CBM, incluindo as formas graves ou refratárias. A dose recomendada da suspensão oral de PCZ em pacientes com CBM é 800 $\mathrm{mg} /$ dia dividido em duas doses durante a terapia. Além disso, este antifúngico é caracterizado por apresentar melhores dados farmacodinâmico e farmacocinética do que o ITZ ${ }^{(4)}$. O VCZ está disponível no mercado na forma de comprimidos revestidos e suspensão oral ${ }^{(6)}$. 
Outro fator importante durante o tratamento é a utilização de associações terapêuticas para casos de resistência ${ }^{(16)}$. A combinação terapêutica pode ser uma alternativa para aumentar a efetividade de cada antifúngicos e melhorar a eficácia do tratamento utilizando doses menores, especialmente em tratamentos sem resposta ao antifúngicos como monoterapia ${ }^{(7,31)}$.

Em nossa pesquisa observamos que associação entre ITZ e TBF são as mais recomendadas para pacientes com lesões refratárias demonstrando bom sinergismo entre estes fármacos. Tal fato pode ser justificado por apresentarem ação em diferentes pontos da mesma via da biossíntese do ergosterol fúngico com a TBF agindo na inibição da enzima escaleno epoxidase e o ITZ na inibição 14- $\alpha$-demetilase ${ }^{(15)}$. ANF + 5-FLU foi a segunda associação mais observada na literatura. ANF-B é considerada ineficaz como monoterapia, mas em combinação com a 5-FLU tem demonstrado eficácia em estudo in vivo ${ }^{(8,16)}$.

Por último, associação do ITZ + 5-FLU foi utilizado em um pequeno número de pacientes, mas demonstrou eficácia em formas graves de micoses subcutâneas. Os dados farmacológicos mostraram um efeito aditivo contra fungos onde a 5-FLU atua na membrana celular e interfere na síntese do DNA e ITZ atuam também membrana celular, inibindo a síntese de ergosterol ${ }^{(8,23)}$. Apesar de um número insuficiente de casos para uma comparação, a terapia combinada com esses dois medicamentos pode ser uma opção nos casos graves ${ }^{(6,24)}$.

\section{CONCLUSÃO}

CBM é uma doença fúngica negligenciada e extremamente difícil tratar, especialmente nas formas clínicas mais graves. Neste estudo foi possível identificar a partir da análise na literatura os fármacos mais utilizados na farmacoterapia da CBM como ITZ, TBF, PCZ e VCZ. Concluímos que o ITZ entre os antifúngicos estudados é o mais eficaz tanto em teste de suscetibilidade in vitro como em situações clínicas. A interação medicamentosa entre ITZ+ TBF é a mais eficiente entre os artigos avaliados na literatura, tornando uma alternativa terapêutica para o tratamento.

\section{REFERÊNCIAS BIBLIOGÁFICAS}

1.Queiroz-Telles F, Nucci M, Colombo AL, Tobón A, Restrepo A. Mycoses of implantation in Latin America: an overview of epidemiology, clinical manifestations, diagnosis and treatment. Med Mycol. 2011 Apr;49(3):225-36. doi: 10.3109/13693786.2010.539631. Epub 2010 Dec 3. PMID: 21128710. 
2. de Sousa Mda G, Belda W Jr, Spina R, Lota PR, Valente NS, Brown GD, Criado PR, Benard G. Topical application of imiquimod as a treatment for chromoblastomycosis. Clin Infect Dis. 2014 Jun;58(12):1734-7. doi: 10.1093/cid/ciu168. Epub 2014 Mar 14. PMID: 24633683; PMCID: PMC4036686.

3.Chaidaroon W, Tananuvat N, Chavengsaksongkram P, Vanittanakom N. Corneal Chromoblastomycosis Caused by Fonsecaea pedrosoi. Case Rep Ophthalmol. 2015 Feb 27;6(1):82-7. doi: 10.1159/000380759. PMID: 25873892 ; PMCID: PMC4376933.

4. Sienkiewicz BM, Łapiński Ł, Wiela-Hojeńska A. Comparison of clinical pharmacology of voriconazole and posaconazole. Contemp Oncol (Pozn). 2016;20(5):365-373. doi: 10.5114/wo.2016.64594. Epub 2016 Dec 20. PMID: 28373817; PMCID: PMC5371702.

5. Correia RT, Valente NY, Criado PR, Martins JE. Chromoblastomycosis: study of 27 cases and review of medical literature. An Bras Dermatol. 2010 Jul-Aug;85(4):448-54. English, Portuguese. doi: 10.1590/s0365-05962010000400005. PMID: 20944904.

6. Agarwal R, Singh G, Ghosh A, Verma KK, Pandey M, Xess I. Chromoblastomycosis in India: Review of 169 cases. PLoS Negl Trop Dis. 2017 Aug 3;11(8):e0005534. doi: 10.1371/journal.pntd.0005534. PMID: 28771470; PMCID: PMC5542425.

7. Deng S, Tsui CK, Gerrits van den Ende AH, Yang L, Najafzadeh MJ, Badali H, Li R, Hagen F, Meis JF, Sun J, Dolatabadi S, Papierok B, Pan W, de Hoog GS, Liao W. Global Spread of Human Chromoblastomycosis Is Driven by Recombinant Cladophialophora carrionii and Predominantly Clonal Fonsecaea Species. PLoS Negl Trop Dis. 2015 Oct 23;9(10):e0004004. doi: 10.1371/journal.pntd.0004004. PMID: 26496430; PMCID: PMC4619687.

8. Brito AC, Bittencourt MJS. Chromoblastomycosis: an etiological, epidemiological, clinical, diagnostic, and treatment update. An Bras Dermatol. 2018 Jul-Aug;93(4):495-506. doi: 10.1590/abd1806-4841.20187321. PMID: 30066754; PMCID: PMC6063100.

9. Lima AM, Sacht GL, Paula LZ, Aseka GK, Goetz HS, Gheller MF, Torraca PF. Response of chromoblastomycosis to voriconazole. An Bras Dermatol. 2016 Sep-Oct;91(5):679-681. doi: 10.1590/abd1806-4841.20165142. PMID: 27828652; PMCID: PMC5087237

10. Lyon JP, Pedroso e Silva Azevedo Cde M, Moreira LM, de Lima CJ, de Resende MA. Photodynamic antifungal therapy against chromoblastomycosis. Mycopathologia. 2011 Oct;172(4):293-7. doi: 10.1007/s11046-011-9434-6. Epub 2011 Jun 4. PMID: 21643843.

11. Mouchalouat Mde F, Gutierrez Galhardo MC, Zancopé-Oliveira RM, Monteiro Fialho PC, de Oliveira Coelho JM, Silva Tavares PM, Francesconi do Valle AC. Chromoblastomycosis: a clinical and molecular study of 18 cases in Rio de Janeiro, Brazil. Int J Dermatol. 2011 Aug;50(8):981-6. doi: 10.1111/j.1365-4632.2010.04729. x. PMID: 21781072.

12. Silva JP, de Souza W, Rozental S. Chromoblastomycosis: a retrospective study of 325 cases on Amazonic Region (Brazil). Mycopathologia. 1998-1999;143(3):171-5. doi: 10.1023/a:1006957415346. PMID: 10353215. 
13. Krzyściak PM, Pindycka-Piaszczyńska M, Piaszczyński M. Chromoblastomycosis. Postepy Dermatol Alergol. 2014 Oct;31(5):310-21. doi: 10.5114/pdia.2014.40949. Epub 2014 Oct 22. PMID: 25395928; PMCID: PMC4221348.

14. Shen XC, Dai XN, Xie ZM, Li P, Lu S, Li JH, Zhang Y, Ye XD. A Case of Chromoblastomycosis Caused by Fonsecaea pedrosoi Successfully Treated by Oral Itraconazole Together with Terbinafine. Dermatol Ther (Heidelb). 2020 Apr;10(2):321-327. doi: 10.1007/s13555-020-00358-y. Epub 2020 Feb 21. PMID: 32086748; PMCID: PMC7090106.

15. Zhang J, Wu X, Li M, Huang J, Yin S, Huang H, Lu C, Xi L. Synergistic effect of terbinafine and amphotericin B in killing Fonsecaea nubica in vitro and in vivo. Rev Inst Med Trop Sao Paulo. 2019 Jun 19;61:e31. doi: 10.1590/S1678-9946201961031. PMID: 31241660; PMCID: PMC6592013.

16. Queiroz-Telles F, de Hoog S, Santos DW, Salgado CG, Vicente VA, Bonifaz A, Roilides E, Xi L, Azevedo CM, da Silva MB, Pana ZD, Colombo AL, Walsh TJ. Chromoblastomycosis. Clin Microbiol Rev. 2017 Jan;30(1):233-276. doi: 10.1128/CMR.00032-16. PMID: 27856522; PMCID: PMC5217794.

17. Lupi O, Tyring SK, McGinnis MR. Tropical dermatology: fungal tropical diseases. J Am Acad Dermatol. 2005 Dec;53(6):931-51, quiz 952-4. doi: 10.1016/j.jaad.2004.10.883. PMID: 16310053.

18. López Martínez R, Méndez Tovar LJ. Chromoblastomycosis. Clin Dermatol. 2007 MarApr;25(2):188-94. doi: 10.1016/j.clindermatol.2006.05.007. PMID: 17350498.

19. Salgado CG, Silva JP, Silva MB, Costa PF, Salgado UI. Cutaneous diffuse chromoblastomycosis. The Lancet Infectious Diseases. 2005 Aug; 8(5):528. doi:10.1016/S1473-3099(05)70195-X.

20. Salgado CG, Silva MB, Yamano SS, Salgado UI, Diniz JAP, Silva JP. Cutaneous localized annular chromoblastomycosis. J Cutan Pathol. 2009 Feb;36(2):257-261. doi: 10.1111/j.16000560.2008.01025.x. PMID: 205816238.

21. Carrasco-Zuber JE, Navarrete-Dechent C, Bonifaz A, Fich F, Vial-Letelier V, BerroetaMauriziano D. Cutaneous Involvement in the Deep Mycoses: A Literature Review. Part ISubcutaneous Mycoses. Actas Dermosifiliogr. 2016 Dec;107(10):806-815. English, Spanish. doi: 10.1016/j.ad.2016.05.017. Epub 2016 Jun 30. PMID: 27374381.

22. Poirriez J, Breuillard F, Francois N, Fruit J, Sendid B, Gross S, Dei-Cas E. A case of chromomycosis treated by a combination of cryotherapy, shaving, oral 5-fluorocytosine, and oral amphotericin B. Am J Trop Med Hyg. 2000 Jul-Aug;63(1-2):61-3. doi: 10.4269/ajtmh.2000.63.61. PMID: 11357997.

23. Queiroz-Telles F. Cromoblastomicosis: A Neglected Tropical Disease. Rev Inst Med Trop Sao Paulo. 2015 Sep;57 Suppl 19(Suppl 19):46-50. doi: 10.1590/S0036-46652015000700009. PMID: 26465369; PMCID: PMC4711190. 
24. Coelho RA, Brito-Santos F, Figueiredo-Carvalho MHG, Silva JVDS, Gutierrez-Galhardo MC, do Valle ACF, Zancopé-Oliveira RM, Trilles L, Meyer W, Freitas DFS, Almeida-Paes R. Molecular identification and antifungal susceptibility profiles of clinical strains of Fonsecaea spp. isolated from patients with chromoblastomycosis in Rio de Janeiro, Brazil. PLoS Negl Trop Dis. 2018 Jul 26;12(7):e0006675. doi: 10.1371/journal.pntd.0006675. PMID: 30048450; PMCID: PMC6080783.

25. Sophie B, Coralie Z, Mai Ba H, Annie L, Dea GH, Liliane L, Arezki I. First case of chromoblastomycosis from Bangladesh. Med Mycol Case Rep. 2015 Sep 2; 10:1-3. doi: 10.1016/j.mmcr.2015.08.001. Erratum in: Med Mycol Case Rep. 2016 Mar; 11:27. PMID: 26484011 ; PMCID: PMC4573089.

26. Rios JE, Paiva CB, Paula GM, Figueiredo WR, Arantes JC, Almeida FM, Limongi RM. Chromomycosis, an unusual cause of cicatricial ectropion: a case report. Arq Bras Oftalmol. 2017 Jan-Feb;80(1):46-48. doi: 10.5935/0004-2749.20170012. PMID: 28380102.

27. Ventura-Flores R, Failoc-Rojas V, Silva-Díaz H. Cromoblastomicosis: características clínicas y microbiológicas de una enfermedad desatendida [Chromoblastomycosis: clinical and microbiological characteristics of a neglected disease]. Rev Chilena Infectol. 2017 Aug;34(4):404-407. Spanish. doi: 10.4067/s0716-10182017000400404. PMID: 29165523.

28. Tourret J, Benabdellah N, Drouin S, Charlotte F, Rottembourg J, Arzouk N, Fekkar A, Barrou B. Unique case report of a chromomycosis and Listeria in soft tissue and cerebellar abscesses after kidney transplantation. BMC Infect Dis. 2017 Apr 20;17(1):288. doi: 10.1186/s12879-017-2386-9. PMID: 28427354; PMCID: PMC5397669.

29. Bao F, Wang Q, Yu C, Shang P, Sun L, Zhou G, Wu M, Zhang F. Case Report: Successful Treatment of Chromoblastomycosis Caused by Fonsecaea monophora in a Patient with Psoriasis Using Itraconazole and Acitretin. Am J Trop Med Hyg. 2018 Jul;99(1):124-126. doi: 10.4269/ajtmh.17-0579. Epub 2018 May 17. PMID: 29785921 ; PMCID: PMC6085797.

30. Heidrich D, González GM, Pagani DM, Ramírez-Castrillón M, Scroferneker ML. Chromoblastomycosis caused by Rhinocladiella similis: Case report. Med Mycol Case Rep. 2017 Apr 7; 16:25-27. doi: 10.1016/j.mmcr.2017.04.002. PMID: 28458993; PMCID: PMC5397130.

31. Chowdhary A, Meis JF, Guarro J, de Hoog GS, Kathuria S, Arendrup MC, Arikan-Akdagli ESCMID and ECMM joint clinical guidelines for the diagnosis and management of systemic phaeohyphomycosis: diseases caused by black fungi.S, Akova M, Boekhout T, Caira M, Guinea J, Chakrabarti A, Dannaoui E, van Diepeningen A, Freiberger T, Groll AH, Hope WW, Johnson E, Lackner M, Lagrou K, Lanternier F, Lass-Flörl C, Lortholary O, Meletiadis J, Muñoz P, Pagano L, Petrikkos G, Richardson MD, Roilides E, Skiada A, Tortorano AM, Ullmann AJ, Verweij PE, Cornely OA, Cuenca-Estrella M; European Society of Clinical Microbiology and Infectious Diseases Fungal Infection Study Group; European Confederation of Medical Mycology. Clin Microbiol Infect. 2014 Apr;20 Suppl 3:47-75. doi: 10.1111/1469-0691.12515. PMID: 24483780. 